\title{
Application of onion-like carbon to micro and nanotribology
}

\author{
N. Matsumoto, L. Joly-Pottuz, H. Kinoshita, N. Ohmae * \\ Faculty of Engineering, Kobe University, 1-1 Rokkodai, Nada, Kobe 657-8501, Japan
}

The tribological properties of onion-like carbon (OLC) have been investigated on a nanometer scale using an ultra-high vacuum atomic force microscope. In addition, the tribological properties of OLC added to oil were investigated. A viewpoint of the practical application of OLC as a lubricating additive to oil, under both experimental conditions, OLC showed low friction. In the latter case, OLC transformed into a low friction material with long life, and appears to be a promising material as nanolubricant.

Keywords: Onion-like carbon; Ultrahigh vacuum atomic force microscopy; Micro and nanotribology; Lubricant additive

\section{Introduction}

Onion-like carbon (OLC) is known as a gigantic molecule consisting of concentric multi-layered graphitic spheres. Ugarte first reported the existence of OLC in 1992 [1], and since then the research groups of Kuznetsov and Chhowalla studied the physical and electronic properties of OLC [2-4]. Regarding the tribological properties of OLC, Cabioc'h et al. found that the friction coefficient of OLC on $\mathrm{Si}$ wafer was 0.01 with the applied force of micron Newton [5]. The application of OLC to micro and nanotribology was also discussed in Refs. [6,7]. However, an atomic force microscope (AFM) used in these preliminary studies did not have an atomic resolution due to the operation in an ordinary atmosphere. To avoid adsorption of gases and other contaminations, an AFM which works in ultrahigh vacuum (UHV) is needed.

The objectives of this study were to characterize the friction properties of OLC on a nano-scale using the UHV-AFM and further to investigate the tribological properties of OLC added to a base oil.

\section{Experimental procedures}

OLC was prepared by annealing the diamond nanoparticles (DNP) in vacuum $\left(10^{-4} \mathrm{~Pa}\right)$ at $1600{ }^{\circ} \mathrm{C}$ and $1700^{\circ} \mathrm{C}$. Com-

\footnotetext{
* Corresponding author. Tel./fax: +81 788036111.

E-mail address: ohmae@mech.kobe-u.ac.jp (N. Ohmae).
}

mercially available DNP with an average diameter of 5-10 nm, prepared by a TNT explosion under the absence of oxygen, was used.

The structures and geometrical shapes of OLC were observed by the UHV high resolution electron microscope (HR-TEM) which works in vacuum of $10^{-8} \mathrm{~Pa}$. The study on the transformation of DNP into OLC by electron-beam irradiation was conducted directly inside this UHV HR-TEM [8].

UHV SPM (Omicron UHV STM/AFM) was used to investigate the tribological properties of OLC, and Nanoscope IIIa mainly to observe the surface of AISI 52100 steel on which a small amount of OLC was supplied with polyalphaolefin (PAO) oil and slid against an AISI 52100 ball.

\section{Results and discussion}

\subsection{Isolated $O L C$}

Fig. 1 presents the AFM (a) and lateral force microscope (LFM) (b) images for friction force with OLC on the cleaved highly oriented pyrolytic graphite (HOPG). UHV-AFM resolves individual OLC in both micrographs, despite that agglomerated OLC exists. Better contrast resulted from the LFM image. The friction coefficient of OLC was measured at 0.05 , while that of HOPG at 0.02 . Moreover, the adhesion force of OLC was $27 \mathrm{nN}$ at an applied force of $60 \mathrm{nN}, 2.5$ times larger than that of HOPG. The details of AFM/LFM measurements and force calibrations are described in Ref. [9]. 

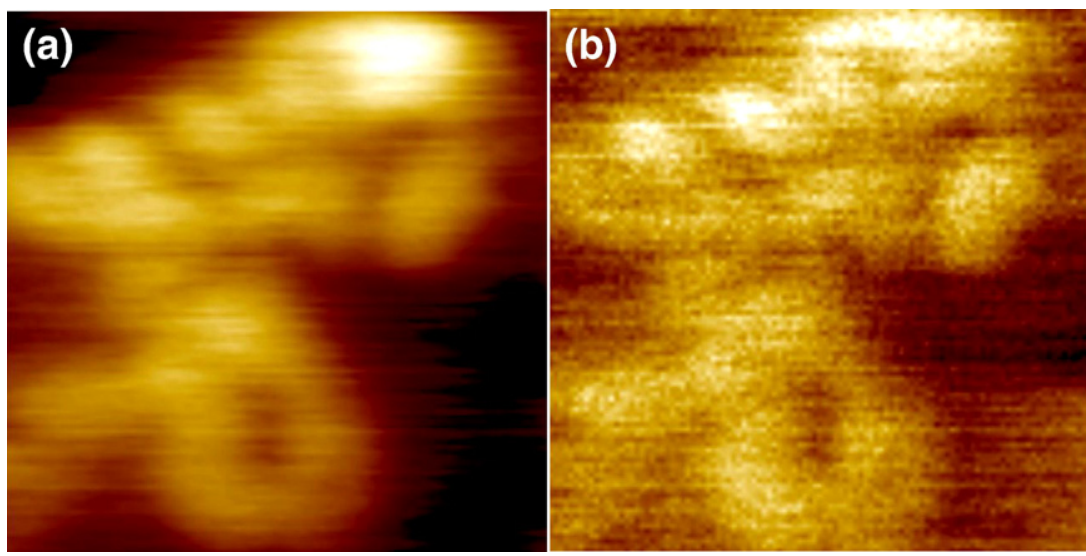

Fig. 1. UHV-AFM image (a) and LFM image (b) showing the morphology and friction of individual OLC on HOPG $(80 \times 80 \mathrm{~nm})$.

\subsection{OLC as nanolubricants}

Carbon onions synthesized at $1700{ }^{\circ} \mathrm{C}$ were dispersed at 0.1 wt. $\%$ in PAO on 52100 steel. Tribological properties were tested at four different contact pressures by changing the load on the ball or the ball diameter. An AISI 52100 ball with a diameter of $6 \mathrm{~mm}$ was used to obtain the Hertzian pressure of $0.83,1.12$ and $1.42 \mathrm{GPa}$. For obtaining $1.72 \mathrm{GPa}$, a ball of $4.5 \mathrm{~mm}$ diameter was used. The sliding speed was $2.5 \mathrm{~mm} / \mathrm{s}$. Fig. 2(a) shows the reduction of friction coefficient as the contact pressure increases. At $0.83 \mathrm{GPa}$ contact pressure, friction initially is high and fluctuates, and then stabilizes as sliding proceeds. At 1.42 and $1.72 \mathrm{GPa}$ contact pressures, the steady state of friction appears after about 100 cycles of sliding, and is very low at 0.07 . This implies that OLC creates low friction material at the contact area and that at high contact pressure this reaction is accelerated. It seems quite interesting to identify this low friction material, and this will be discussed later.

Besides lowering friction, the use of OLC results in lowered wear width of the ball. For the $6 \mathrm{~mm}$ ball, the width of wear scar on the ball was 170,175 and $180 \mu \mathrm{m}$ at $0.83,1.12$ and $1.42 \mathrm{GPa}$ contact pressures, when PAO alone was used as a lubricating oil. For the $4.5 \mathrm{~mm}$ ball at $1.72 \mathrm{GPa}$ contact pressure, the wear scar was $150 \mu \mathrm{m}$. By adding 0.1\% OLC to PAO, the wear scar reduces to $90,115,135$ and $115 \mu \mathrm{m}$, for the four different levels of contact pressure. These values are up to $120 \%$ of calculated Hertzian diameter. This signifies the effectiveness of OLC for anti-wear purpose.

The same experiments as shown in Fig. 2(a) were carried out by using the $1 \mathrm{~mm}$ diameter ball at a contact pressure of 1.46 GPa. No effective reduction in friction resulted (see Fig. 2 (b)). Friction fluctuates substantially after a few hundred cycles of sliding. The reason for this may be due to an insufficient supply of OLC to the contacting interface because of the small contact diameter (approximately $20 \mu \mathrm{m}$ ).

Fig. 3(a) and (b) shows the AFM and LFM images of the surface on which a $6 \mathrm{~mm}$ ball slid at a pressure of $1.42 \mathrm{GPa}$. The surface appears smooth and contains OLC aligned in the sliding direction. It should be noted that the friction is low at the location where OLC exists. For about $5 \mu \mathrm{m} \times 5 \mu \mathrm{m}$ scan, the maximum height of the surface is only $20 \mathrm{~nm}$.
A rough appearance with the maximum height of $70 \mathrm{~nm}$ was observed on the surface on which a $1 \mathrm{~mm}$ ball slid at a pressure of $1.42 \mathrm{GPa}$ (see Fig. 3(c) and (d)). The width of the bands aligned in the horizontal direction is narrow at about $1 \mu \mathrm{m}$, and it is clear that the formation of low friction material is not sufficient in this case.

At higher magnifications of Nanoscope IIIa, the surface of Fig. 3(c) looks rough and discontinuous, as shown in Fig. 3(e). Discontinuity in the vertical direction of individual narrow bands as well as scaly appearance of deformed OLC is observed. After sliding, OLCs are not spherical in shape any more,
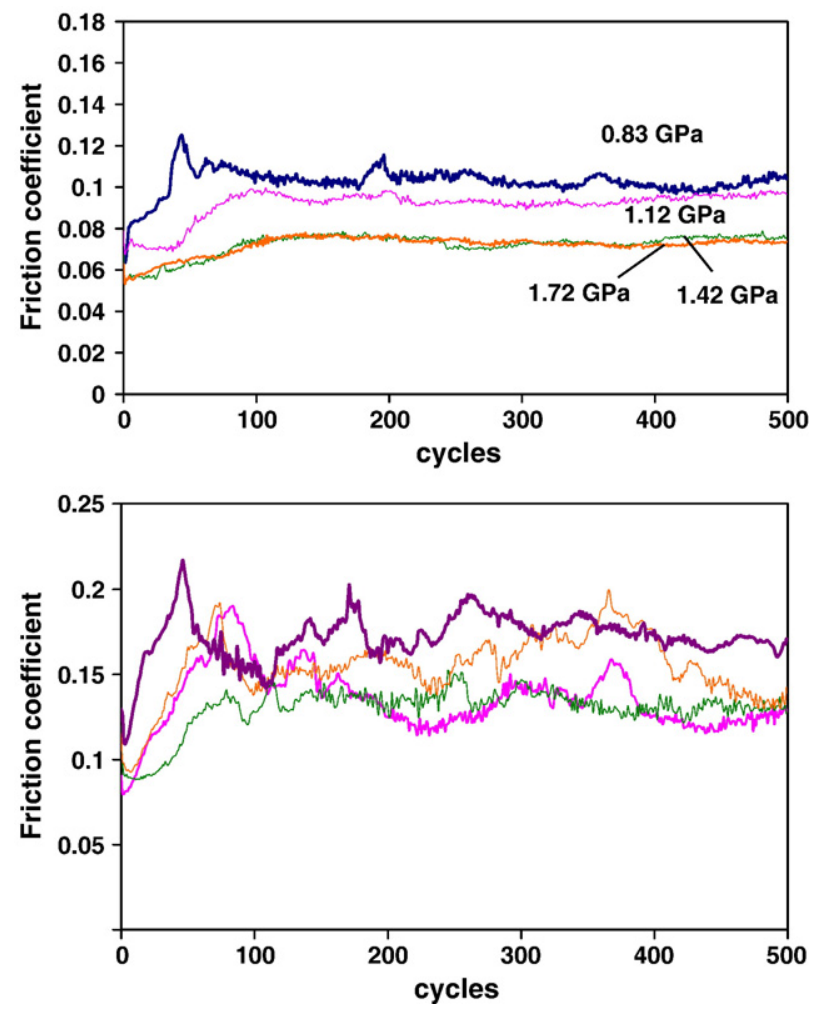

Fig. 2. Reduction of friction by OLC added to PAO, obtained from sliding of $6 \mathrm{~mm}$ and $4.5 \mathrm{~mm}$ diameter AISI balls (a), and insufficient reduction of friction by added OLC resulted from a sliding of $1 \mathrm{~mm}$ diameter AISI ball (b). Four lines indicate repeated test results with the same condition in (b). 


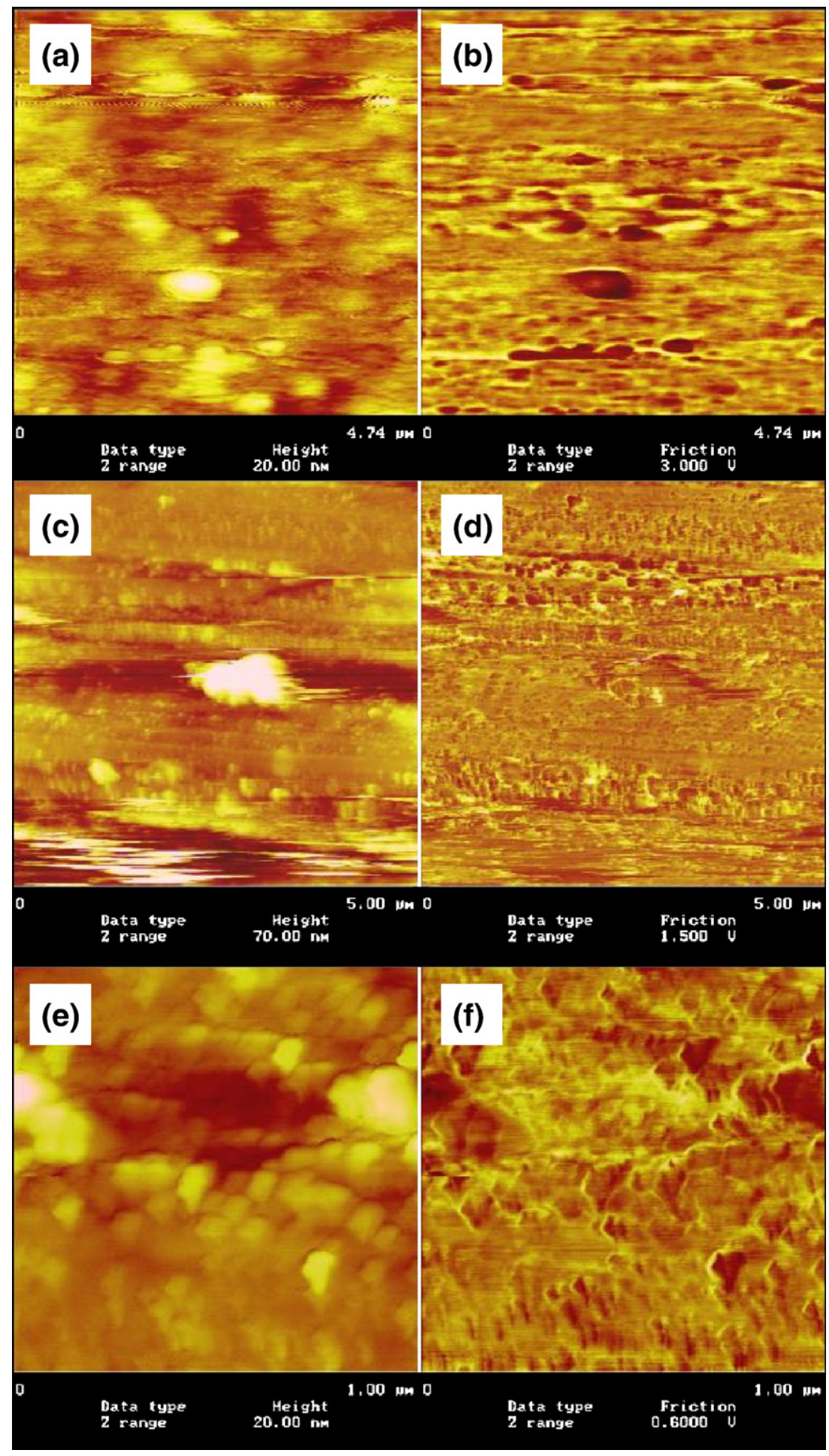

Fig. 3. AFM/LFM images of tribo-transformed film obtained from the sliding shown in Fig. 2(a) with the contact pressure of 1.42 GPa (a and b), and shown in Fig. 2 (b-d). In Fig. 2(c), sufficient formation of tribo-transformed film was not observed. At higher magnification of Fig. 5(c and d), OLC appears scaly (e and f).

but rather smashed. Fig. 3(a) showed that the deformed OLC aligned in the direction of sliding to form a wide and smooth film, while Fig. 3(c) and (e) shows that the transformation into the smooth film is not completed.

The UHV-STM photograph of the surface shown in Fig. 3(a) is indicated in Fig. 4(a) and (b). The smooth band observed in
Fig. 3(a) consists of narrow bands with a width of $0.1 \mu \mathrm{m}$, and this narrow band consists of several to 10 narrower bands (see Fig. 4(a)). Thus the structure of the low friction film shows a fractal feature. The surface is extremely smooth with the height of about $12 \mathrm{~nm}$. The formation of a smooth film consisting of deformed OLC is the strongest reason for low friction shown 


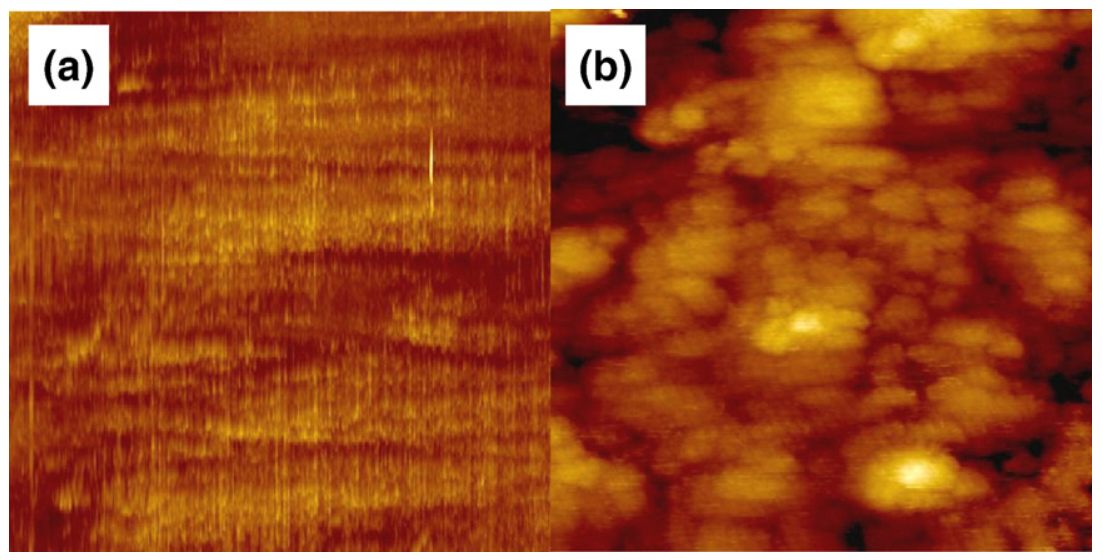

Fig. 4. UHV-STM images of the alignment of deformed OLC $(700 \times 700 \mathrm{~nm})(\mathrm{a})$, and higher magnification $(200 \times 200 \mathrm{~nm})(\mathrm{b})$.

previously in Fig. 2. Aligned narrower bands $0.1 \mu \mathrm{m}$ wide consist of aggregated OLCs. The aggregation of OLCs is made up 10-20 molecules. It is amazing to note that the maximum height in Fig. 4(b) is less than $10 \mathrm{~nm}$, i.e., smaller than the average diameter of OLC. It is likely that OLCs were fractured during the processes of alignment, since none of the large OLCs existed in the film.

As has been discussed, lowered friction by adding OLC to PAO relates clearly to the formation of a very smooth surface film consisting of deformed OLC. Fig. 5 demonstrates the UHV-STM taken with an atomic resolution. Within a smooth flat surface film, over 20 carbon atoms are buried. These atoms originate in the fractured OLC, because the atoms are distributed in the area of a few nanometers, far smaller than the whole OLC. It appears that the arrangement of atom sites curves slightly. The distance between the curved array is $0.34 \mathrm{~nm}$, which corresponds to the spacing of basal planes. Therefore, Fig. 5 may present the cross-section of OLC. The estimated diameter from the curvature is $11 \mathrm{~nm}$, consistent with that of OLC. One thing puzzling to us is the distance between

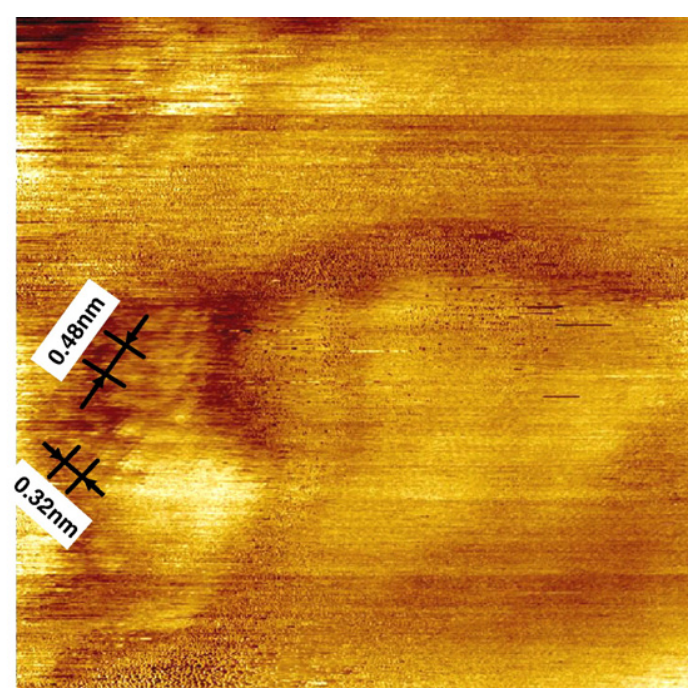

Fig. 5. UHV-STM image indicating the fractured piece of OLC in the smooth tribo-transformed film. the atomic sites along the periphery, the value being $0.48 \mathrm{~nm}$, double of the atomic distance of the basal plane. There has been no STM showing the prism plane of graphite, and it is not clear, at present, whether or not the atoms on the prism plane are imaged by STM.

\section{Conclusion}

Not only the friction property of OLC on a molecular scale but also the use of OLC as a lubricant additive has been presented. The friction coefficient of OLC in UHV was 0.05 , low enough to be used in vacuum or in space environment. AFM and STM revealed the characteristic structure of low friction film (as low as 0.07 in PAO), tribologically transformed from the OLC placed on 52100 steel. Therefore, the application of OLC as a nanolubricant appears very promising.

\section{Acknowledgement}

This work was partially supported by the Grant-in-Aid for scientific Research (A) from the Ministry of Education, Culture, Sport, Science and Technology.

\section{References}

[1] D. Ugarte, Nature 359 (1993) 707.

[2] V.L. Kuznetsov, A.L. Chuvilin, Y.V. Butenko, I.Y. Mal'kov, V.M. Tikov, Chem. Phys. Lett. 222 (1994) 343.

[3] V.L. Kuznetsov, Y.V. Butenko, A.L. Chuvilin, A.I. Romanenko, A.V. Okotrub, Chem. Phys. Lett. 336 (2001) 397.

[4] N. Sano, H. Wang, I. Alexandrou, M. Chhowalla, K.B.K. Teo, K. Iimura, J. Appl. Phys. 92 (2002) 278

[5] T. Cabioc'h, E. Thune, J.P. Riviere, S. Camelio, J.C. Girard, P. Guerin, J. Appl. Phys. 91 (2002) 1560.

[6] N. Ohmae, J.M. Martin, S. Mori, Micro and Nanotribology, ASME Press, NY, 2005, p. 88

[7] N. Ohmae, et al., Synopses of International Tribology Conference, vol. 28, Kobe, 2005

[8] J. Hiraki, H. Mori, E. Taguchi, H. Yasuda, H. Kinoshita, N. Ohmae, Appl. Phys. Lett. 86 (2005) 223101.

[9] N. Matsumoto, L. Joly-Pottuz, H. Kinoshita, N. Ohmae, Proceedings of the International Tribology Conference Austrib 06, 303. 